\title{
Consequences of cellular cholesterol accumulation: basic concepts and physiological implications
}

\author{
Ira Tabas \\ Department of Medicine, and Department of Anatomy and Cell Biology, Columbia University, New York, New York, USA \\ J. Clin. Invest. 110:905-911 (2002). doi:10.1172/JCI200216452.
}

The cells of most organs and tissues satisfy their requirements for membrane cholesterol via endogenous cholesterol biosynthesis (1). Many cell types, however, have acquired mechanisms to internalize exogenous sources of cholesterol, usually in the form of plasma-derived lipoproteins (2). Examples include steroid-synthesizing cells, hepatocytes, and macrophages and smooth muscle cells in atherosclerotic lesions, often referred to as foam cells. In the case of steroidogenic cells, the internalization of lipoprotein-cholesterol represents a physiological process that provides cells with precursor cholesterol stores, to be used for "acute" steroid hormone production (3). Hepatocyte lipoprotein uptake mediates the clearance of various classes of plasma lipoproteins (1), which can lead to whole-body elimination of excess dietderived cholesterol in the bile, a process known as reverse cholesterol transport (Tall, this Perspective series, ref. 4). The uptake of arterial-wall lipoproteins by macrophages and smooth muscle cells may be a type of physiological scavenging response that initially helps rid the endothelium of potentially harmful lipoprotein material (5). As will be discussed below, however, this cellular process eventually contributes to the progression and complications of atherosclerotic vascular disease.

Cells that rely totally or mostly on endogenous cholesterol synthesis cannot accumulate excess endogenous cholesterol because of homeostatic regulation at multiple steps in the cholesterol biosynthetic pathway (6). Cells that internalize exogenous cholesterol also repress endogenous cholesterol biosynthesis and LDL receptor expression in response to cholesterol loading. Furthermore, these cells have evolved other mechanisms to prevent the accumulation of excess unesterified, or "free," cholesterol (FC). One mechanism is cholesterol esterification, which is mediated by the microsomal enzyme acyl-coenzyme A:cholesterol acyltransferase (ACAT) (7)

\footnotetext{
Address correspondence to: Ira Tabas, Department of Medicine, Columbia University, 630 West 168th Street, New York, New York 10032, USA. Phone: (212) 305-9430; Fax: (212) 305-4834;

E-mail: iat1@columbia.edu.

Conflict of interest: No conflict of interest has been declared. Nonstandard abbreviations used: free cholesterol (FC); acylcoenzyme A:cholesterol acyltransferase (ACAT); cholesteryl ester (CE); Niemann-Pick C protein 1 (npc1); endoplasmic reticulum (ER); phosphatidylcholine (PC); CTP:phosphocholine cytidylyltransferase (CT).
}

(Figure 1). The two forms, ACAT-1 and ACAT-2, differ in their sites of expression, with macrophages and most other cell types expressing ACAT-1. In humans, intestinal epithelial cells, but not hepatocytes, selectively express ACAT-2; mice, in contrast, express ACAT-2 in both of these cell types (7). Another important protective mechanism against FC accumulation is cellular efflux of cholesterol and certain cholesterol-derived oxysterols (Tall, this Perspective series, ref. 4; Björkhem, this series, ref. 8) (Figure 1). In addition, some of the physiological pathways described above, such as steroid and bile acid biosynthesis, may help limit the accumulation of intracellular FC in steroidogenic cells and hepatocytes, respectively (2).

The evolution of multiple pathways to prevent intracellular FC accumulation reflects the toxic effects of excess cellular FC (9). In this article, I first discuss possible mechanisms of FC-induced cytotoxicity. I then turn to the development of advanced atherosclerotic lesions, a critical pathophysiological scenario in which macrophages accumulate excess FC. This scenario provides a valuable model to study mechanisms of FC accumulation, the adaptive processes that cells employ to help protect themselves from excess FC, and pathways and consequences of FC-induced cell death. The conversion of the lesional macrophage from a cholesteryl ester-laden (CE-laden) foam cell into an FCloaded cell may, I argue, represent a critical turning point in the progression of the atherosclerotic lesion.

\section{Mechanisms of FC-induced cytotoxicity}

A physiological $\mathrm{FC} /$ phospholipid ratio in cellular membranes is necessary to maintain proper membrane fluidity, or more precisely, a proper range of membrane fluidities (10). The degree of saturation of the fatty acyl moieties of membrane phospholipids is the major determinant of the fluidity of lateral membrane domains, which consist of well-packed, detergentresistant liquid-ordered rafts and more fluid, detergent-soluble liquid-crystalline regions (Simons and Ehehalt, this Perspective series, ref. 11; Maxfield and Wüstner, this series, ref. 12). Nonetheless, the interaction of the hydrophobic rings of cholesterol with these fatty acyl chains has important effects (Tabas, Series Introduction, ref. 13). In particular, the ability of cholesterol to pack tightly with saturated fatty acyl 


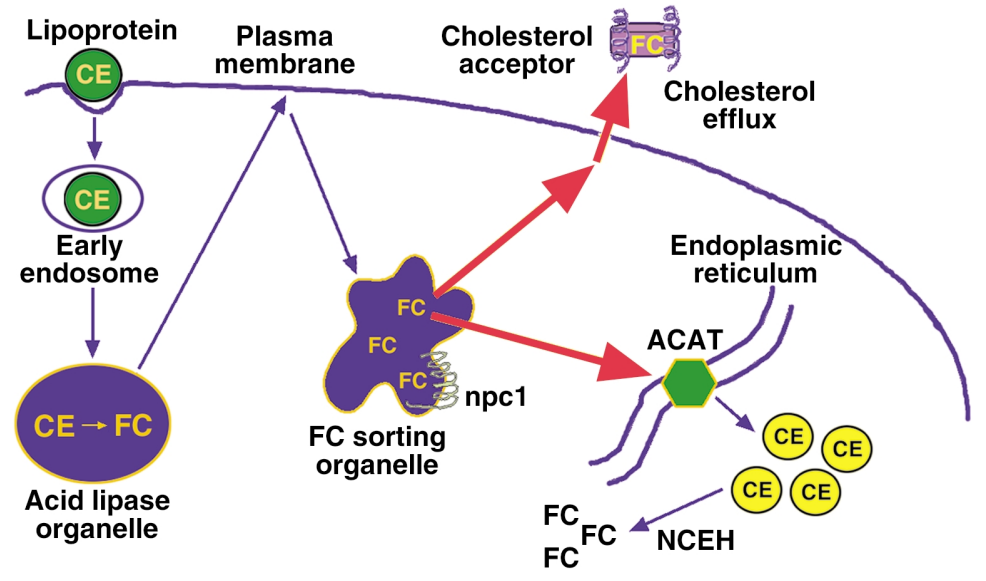

Figure 1

Mechanisms that protect cells from excess accumulation of FC. Lipoprotein-derived cholesterol is distributed to peripheral cellular sites from a putative FC-sorting organelle, which may be a type of late endosome. The npc1 protein is depicted in this organelle as one of the molecules that are known to influence cholesterol trafficking. The cholesterol trafficking itineraries depicted here include transport to ACAT in the endoplasmic reticulum, leading to cholesterol esterification, and to sites of cholesterol efflux in the plasma membrane, leading to removal of cholesterol if appropriate extracellular cholesterol acceptors are present. Not depicted here are those pathways that downregulate the LDL receptor and cholesterol biosynthetic enzymes and those pathways in specialized cells that lead to the metabolism of cholesterol to other molecules, like bile acids and steroid hormones. As described in the text, FC accumulation can occur via inhibition of cholesterol transport to ACAT or to the plasma membrane, or by direct inhibition of ACAT or cholesterol efflux molecules. An increase in neutral cholesteryl esterase (NCEH) activity in the absence of compensatory re-esterification or efflux of cholesterol could also lead to FC accumulation.

groups of membrane phospholipids is critical for the formation of liquid-ordered rafts (10). Thus, cholesterol depletion causes marked disruption of these rafts. However, when the FC/phospholipid ratio rises above a physiological level, the liquid-ordered rafts may become too rigid, and the liquid-crystalline domains may begin to lose their fluidity. These events adversely affect certain integral membrane proteins that require conformational freedom for proper function and that can be inhibited by a high FC/phospholipid ratio (14). Such proteins include plasma membrane constituents like $\mathrm{Na}^{+}-\mathrm{K}^{+}$ATPase, adenylate cyclase, alkaline phosphatase, rhodopsin, and transporters for glucose, organic anions, and thymidine. Similar observations have been made with proteins residing in internal membranes, such as the $\mathrm{Na}^{+}-\mathrm{Ca}^{2+}$ transporter in the sarcoplasmic reticulum of cardiac muscle, the ATPADP transporter in the inner mitochondrial membrane, and UDP-glucuronyltransferase in liver microsomes (14). Interestingly, inhibition of ACAT activity in Chinese hamster ovary cells transfected with amyloid precursor protein blocks the generation of amyloid $\beta$-peptide (15). Although the mechanism of this effect is not yet known, one possibility is that the conformation of the amyloid precursor or the $\beta$-peptide-generating proteases is altered by an increase in the local FC/phospholipid ratio (Simons and Ehehalt, this series, ref. 11). High FC levels might therefore be proposed to kill cells in part by inhibiting one or more integral membrane proteins whose function is blocked or altered under conditions of high membrane rigidity. This and several other models for FC-induced cell death, discussed below, are summarized in Table 1.

Excess membrane cholesterol may also disrupt the function of signaling proteins that reside in membrane domains. For example, when human neutrophils with a normal plasma membrane cholesterol content are stimulated to migrate by exposure to chemokines, the actin-signaling protein Rac is recruited to detergent-sensitive non-raft membrane domains in leading lamella. However, when the plasma membrane of these cells are overloaded with cholesterol, $\mathrm{Rac}$ is recruited to the entire circumference of the plasma membrane, lamellar extension is nonvectorial, and neutrophil migration does not occur (L.M. Pierini and F.R. Maxfield, personal communication). One interpretation of these data is that excess plasma membrane cholesterol disrupts the function of certain signaling molecules that normally reside in non-raft domains. Experiments in vitro with model membranes suggest that, in membranes already enriched in sphingolipids (like those that exist in several types of epithelial cells), increasing the FC concentration even modestly above the physiological concentration can actually suppress the formation of membrane domains (16). Other mechanisms of cellular toxicity associated with FC accumulation include intracellular cholesterol crystallization, oxysterol formation (Björkhem, this series, ref. 8), and triggering of apoptotic signaling pathways (9). Needle-shaped cholesterol crystals form when the FC/phospholipid ratio reaches a very high level. Although typically seen in extracellular regions of advanced atherosclerotic lesions, intracellular cholesterol crystals have been observed both in cultured macrophages overloaded with cholesterol and in foam cells isolated directly from human coronary atherosclerotic lesions $(17,18)$. Intracellular cholesterol crystals can probably damage cells by physically disrupting the integrity of intracellular structures. Excess intracellular FC accumulation can also promote the oxidation of cholesterol to oxysterols, some of which may be cytotoxic (19). Finally, FC overloading of macrophages can trigger a series of apoptotic pathways (20-22), as discussed below.

\section{Accumulation of FC in macrophages during atherogenesis}

The hallmark of the early atherosclerotic lesion is the CE-laden macrophage foam cell (23). Remarkably, in the face of extremely high levels of CE, these early lesional cells maintain an FC content not markedly different from non-foam cell macrophages. However, lipid assays of lesional material from various stages of human and animal atheromata reveal a steady increase 
in FC content and a steady decrease in CE content as the lesions become more advanced (24). While a portion of this trend reflects extracellular events, analysis of foam cells isolated from advanced lesions clearly demonstrates an increase in macrophage FC content (9). As discussed below, cultured macrophage models of FC accumulation have revealed fascinating cellular responses to FC accumulation, as well as end-stage consequences of FC loading that are relevant to the progression and complications of atherosclerosis.

\section{Mechanisms of FC accumulation}

Macrophages are normally protected from the accumulation of excess FC by ACAT-1-mediated esterification and by cholesterol efflux (2) (Figure 1). In addition, the hydrolysis of stored CE by neutral CE hydrolase does not usually exceed a cell's capacity to export or reesterify this pool of cholesterol. Thus, the progressive accumulation of FC by lesional macrophages might be explained by failure of one or more of these protective mechanisms as the atherosclerotic lesion progresses.

Both cholesterol efflux and cholesterol esterification would be adversely affected by disruption of intracellular cholesterol transport. Cholesterol that enters cells via internalization of lipoproteins is initially targeted to an organelle, probably a type of late endosome, that then distributes this cholesterol to the plasma membrane for efflux and the endoplasmic reticulum (ER) for esterification $(2,25,26)$. Peripheral cholesterol transport is an energy-dependent vesicular process and involves molecules such as Niemann-Pick C protein 1 (npc1) and npc2 (HE1) and the lipids lysobisphosphatidic acid and sphingomyelin $(12,25)$. Disturbance of cellular membrane vesiculation, depletion of cellular ATP stores, or disruption of the function of these molecules could block FC efflux and esterification, thus favoring FC accumulation. Indeed, peripheral FC trafficking is inhibited in cultured macrophages incubated with oxidized LDL, a form of modified LDL found in atherosclerotic lesions. This response may be related to inhibition by oxysterols of lysosomal sphingomyelinase (27), leading to sphingomyelin accumulation. Excess intracellular sphingomyelin, in turn, disrupts normal peripheral cholesterol distribution by an unknown mechanism (28). Interestingly, experiments in cultured cells have revealed that lysosomal FC accumulation itself inhibits lysosomal sphingomyelinase activity (29). Thus, if cellular cholesterol influx begins to exceed the capacity of lysosomes to transport FC, an initially modest accumulation of lysosomal FC may be amplified by subsequent inhibition of lysosomal sphingomyelinase. This mechanism may help explain the finding that cultured macrophages that phagocytose large quantities of $\mathrm{CE}$ droplets accumulate FC in lysosomes (30).

Direct disruption of ACAT- 1 or cellular efflux pathways would also promote FC accumulation. Efflux might be compromised by inhibit-

Table 1 ing ABCA1, SR-BI, or other plasma membrane proteins that mediate cholesterol efflux. Indeed, recent data from our laboratory suggest that excess FC accumulation in macrophages compromises the function of ABCA1, which may then amplify further FC accumulation (31). Efflux may also be blocked if access is lost to plasma-derived cholesterol acceptors like HDL and apoA-1, as may occur for macrophages buried deep in the intima of atherosclerotic lesions. In addition, if cellular cholesterol influx exceeds the capacity of ACAT-1, FC may begin to accumulate in the ER membrane domains where this enzyme resides. Because these membranes are normally cholesterol-poor (32), an FCinduced change in the physical properties of these membranes might compromise ACAT-1 activity (33). According to this model, FC accumulation would be amplified by progressive cholesterol accumulation in ER membranes, leading to further ACAT-1 dysfunction. Finally, efflux pathways may also be inhibited by conversion of cholesterol into oxysterols. While certain oxysterols actually promote sterol removal (e.g., 27hydroxycholesterol in macrophages and bile acids in hepatocytes), other oxysterols (e.g., 25-hydroxycholesterol and 7-ketocholesterol) exacerbate cholesterol accumulation by inhibiting FC efflux or ACAT-1-mediated cholesterol esterification (19).

Recent experiments in cultured macrophages suggest that neutral CE hydrolase activity can further complicate the problem of cellular FC homeostasis in advancing atherogenic lesions. When these cells are incubated for a short period with atherogenic lipoproteins, the CE-rich lipid droplets formed have a high CE content and essentially normal FC content, as expected. After subsequent culture in the absence of lipoproteins, however, CE content drops and FC content rises correspondingly. Collections of FC can be seen in the cytoplasm of these cells by filipin staining, reflecting the ability of neutral CE hydrolase to act on preformed CE droplets and to generate excess FC under at least some physiological conditions (34). Whether this enzyme is also active when cells are continuously exposed to atherogenic lipoproteins remains to be determined. Nonetheless, the action of this hydrolase is likely to contribute to FC accumulation in lesional macrophage foam cells whose FC efflux or reesterification by ACAT- 1 is compromised.

\section{Adaptive processes}

As shown in Figure 2, the responses of macrophages to FC loading can be divided into two phases - an initial

Potential mechanisms of FC-induced cytotoxicity

Event

Loss of membrane fluidity

Disruption of membrane domains

Induction of apoptosis

Intracellular cholesterol crystallization

Formation of toxic oxysterols

? Alteration of gene expression

\section{Consequence}

Dysfunction of integral membrane proteins

Disruption of signaling events

Caspase-mediated cell death

Organelle disruption

? Oxidative damage

? Change in balance of survival vs. death proteins 


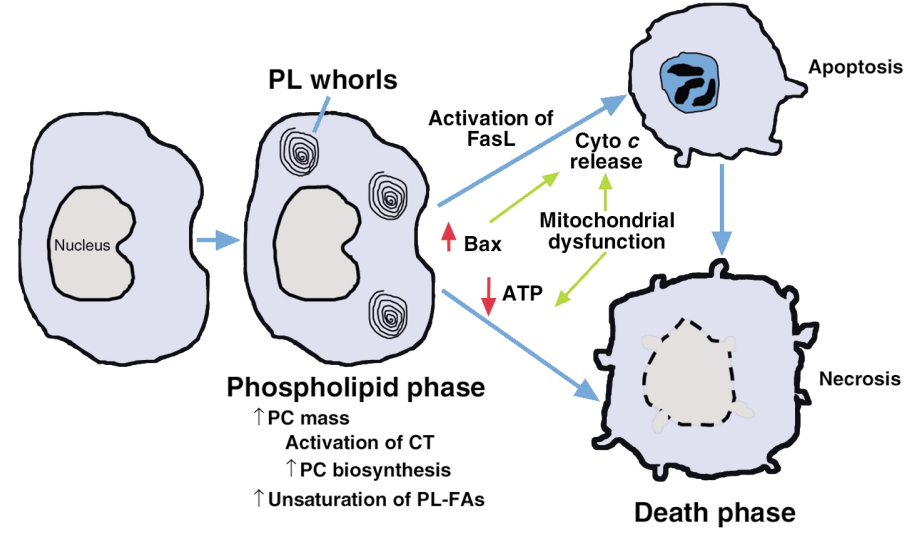

Figure 2

Sequential responses of cultured macrophages to FC loading. In the initial, adaptive phase, CT is activated, leading to increased PC biosynthesis and PC mass and resulting in a normalization of the $\mathrm{FC} /$ phospholipid ratio. In addition, there is an increase in the degree of unsaturation of phospholipid fatty acids (FAs). With continued FC loading, death ensues by both apoptotic and necrotic processes. Apoptosis involves both activation of Fas ligand and release of cytochrome $c$ (cyto $c$ ) from mitochondria, which may be related to the increased levels of mitochondria-associated Bax in FC-loaded macrophages. FC-induced mitochondrial dysfunction also leads to depletion of cellular ATP stores, which could trigger cellular necrosis.

adaptive stage in which phospholipid synthesis increases to offset the harmful effects of increasing FC, and a later stage when these defenses are overcome, leading to cell death. As investigators continue to explore the mechanisms of FC accumulation in vivo, a convenient cell-culture model has enabled the study of both of these stages $(9,35)$. In this model, macrophages are treated with one of several specific inhibitors of ACAT-1, either during or after incubation with atherogenic lipoproteins. Importantly, the perinuclear distribution of filipin-positive FC in the cultured cells mimics the distribution of FC observed in macrophages isolated from atherosclerotic lesions. Moreover, the various effects observed in this model are not seen with ACAT inhibitors in the absence of lipoproteins and are thus dependent on FC loading per se.

One of the earliest responses of macrophages to FC loading is an increase in phospholipid biosynthesis (35). While this response is observed with sphingomyelin and several other classes of phospholipids, the major species produced is phosphatidylcholine (PC). The mechanism involves posttranslational activation of the rate-limiting enzyme in PC biosynthesis, CTP:phosphocholine cytidylyltransferase (CT). Exactly how FC accumulation leads to CT activation is not known, but the process requires dephosphorylation of $\mathrm{CT}$ and possibly one or more $\mathrm{CT}$ regulatory proteins. The twofold increment in PC mass in FC-loaded macrophages is remarkable, and it is reflected by the appearance of phospholipid-containing whorl-like membrane structures in the cells. Importantly, such structures have also been seen in lesional macrophages (36). This observation, together with other reports showing an increase in phospholipid biosynthesis in the cells of atherosclerotic lesions in vivo (37), suggests that the responses of FC-loaded cultured macrophages indeed mimic physiological events.

The central issue to arise from these observations concerned the functional role of this phospholipid response. The hypothesis that increasing PC represents an adaptive response, preventing $\mathrm{FC}$-mediated cytotoxicity by keeping the $\mathrm{FC} /$ phospholipid ratio in check, received direct support from studies conducted with peritoneal macrophages from CT-deficient mice (38). There are two genes that encode CT, CCT $\alpha$ and CCT $\beta$. Macrophages from mice with homozygous disruption of the CCT $\alpha$ gene have intact CT $\beta$ activity and thus retain approximately $20 \%$ of their total CT activity and PC biosynthetic capacity. Importantly, these cells appear healthy when grown under normal culture conditions and are essentially indistinguishable from wildtype macrophages. However, when subjected to an FC load, CT $\alpha$-deficient macrophages cannot mount a substantial PC response, and they succumb to the toxic effects of FC much earlier than do wild-type macrophages. Thus, the ability of FC-loaded macrophages to activate $\mathrm{CT}$ and increase PC biosynthesis helps protect them from FC-induced cytotoxicity.

Ikonen and coworkers (39) recently reported another phospholipid response to FC loading, namely, an increase in plasma membrane phospholipid species with polyunsaturated acyl chains. These studies were conducted with human fibroblasts that had been loaded with FC either by incubation with serum in the face of a mutation that blocks cholesterol esterification or, in wild-type fibroblasts, by acute plasma membrane loading. In both cases, there was a significant increase in the content of polyunsaturated fatty acids in several classes of phospholipids. Although the mechanism of these fatty acid alterations is not known, these changes may represent another adaptive effect to FC loading, because membranes rich in phospholipids with polyunsaturated acyl chains are more resistant to the stiffening effects of cholesterol (40).

\section{FC-induced cell death}

Although cells are clearly capable of adaptive responses to FC loading, these mechanisms eventually fail with prolonged internalization of cholesterol, leading to cell death $(9,41)$ (Figure 2). The basis of adaptive failure is not known, although a decrease in CT activity has been observed before the onset of cellular toxicity (42). CT, like ACAT, is associated with normally cholesterol-poor cellular membranes and thus may become dysfunctional when its lipid microenvironment becomes too rigid.

By morphological criteria, cytotoxic FC-loaded macrophages show signs of both necrosis (e.g., disrupted cellular membranes) and apoptosis (e.g., condensed nuclei) $(9,41)$. By biochemical criteria, apoptosis-associated caspases and their signaling pathways are activated in a portion of the cells (below). In all likelihood, as depicted in Figure 3, some cells in a 
population of FC-loaded macrophages become acutely necrotic due to direct and acute disruptive effects on membrane proteins, whereas others undergo a programmed apoptotic response. Moreover, cells that initially undergo an apoptotic program can subsequently demonstrate morphological signs of necrosis (so-called aponecrosis), perhaps as a result of chronic ATP depletion or failure of neighboring cells to phagocytose the apoptotic bodies (43).

In a typical cell-culture model of FC-loaded macrophages, approximately $25-30 \%$ of the cells show the apoptosis-associated hallmarks of phosphatidylserine externalization and DNA fragmentation, which can be completely prevented by inhibitors of effector caspases (21). Interestingly, partial inhibition of apoptosis is also observed when the Fas receptor signaling pathway is disrupted either by genetic mutations in Fas or its ligand or by use of a blocking anti-Fas ligand antibody. FC loading results in posttranslational activation of cell-surface Fas ligand, either by inducing a conformational change in the molecule or by stimulating its transport from intracellular stores to the plasma membrane (21).

Widespread mitochondrial dysfunction, indicated by a decrease in the mitochondrial transmembrane potential, is also observed in FC-loaded macrophages (22). Furthermore, FC-loaded macrophages show evidence of mitochondrial cytochrome $c$ release and caspase-9 activation. Thus, in addition to involvement of the Fas pathway, a classic mitochondrial pathway of apoptosis is activated in FC-loaded macrophages. Importantly, these events are not mediated by oxysterols, because oxysterol-induced mitochondrial dysfunction and apoptosis are inhibited by the antioxidant glutathione, whereas the death pathways described above are resistant to this compound. The mechanisms by which FC overloading triggers these events is not known, but it appears to be independent of direct effects of FC on the plasma membrane (P.M. Yao and I. Tabas, unpublished data). Intracellular and mitochondrial levels of the proapoptotic protein Bax are increased in FC-loaded macrophages (22), but direct proof for the involvement of Bax is lacking. Other possibilities include direct toxic effects of FC on mitochondrial membranes and activation of a proapoptotic signaling pathway.

An important concept to arise from these cell-culture studies is the importance of intracellular cholesterol trafficking in FC-induced death. Rothblat and colleagues (20) were the first to show that amphipathic amines, which markedly inhibit peripheral cholesterol trafficking, protect FC-loaded macrophages from death. These results suggest that, whether FC triggers death by direct membrane effects or by activation of death-promoting molecules or both, FC must be able to traffic to peripheral sites in the cell to effect cell killing. Our recent data indicate that, whereas FC trafficking to the plasma membrane may be responsible for necrotic-type death, excess FC accumulation in the plasma membrane cannot explain apoptotic death. Moreover, FC-induced apoptosis is blocked by very subtle disruptions of intracellular trafficking, such as occur in macrophages from heterozygous NPC mice or in macrophages treated with very-low-dose amphipathic amines (ref. 44; and P.M. Yao and I. Tabas, unpublished data). Thus, the peripheral apoptosissensing mechanisms appear to be quite sensitive to FC. Clearly, an important goal of further research in this area is to define the molecular mechanisms that underlie the relationship between intracellular cholesterol trafficking and apoptosis in FC-loaded cells.

\section{Relevance to atherosclerotic vascular disease}

The presence of apoptotic and necrotic macrophages in atherosclerotic lesions has been well documented in many human and animal studies $(45,46)$. Among the potential causes of lesional macrophage death, FCinduced toxicity needs serious consideration, because macrophages from advanced lesions are known to be loaded with FC, a potent inducer of macrophage death (9). The functional significance of the cell death pathways depicted in Figure 3 remains uncertain. On the one hand, assuming harmless disposal of apoptotic bodies by neighboring phagocytes, macrophage apoptosis may limit the number of intimal cells in a physiologically "safe" manner that avoids inducing local

\section{Figure 3}

Hypothetical model relating FC loading of lesional macrophages (M $\phi s$ ) to acute events in advanced atheromata. Progressive FC loading of lesional macrophages leads to a series of phospholipid-related adaptive responses, as described in the text. These adaptive responses eventually fail, leading to macrophage death (see Figure 2). On the one hand, macrophage death may contribute to plaque instability by promoting lesional necrosis. On the other hand, safe disposal of apoptotic macrophages could decrease the number of macrophages that secrete matrix metalloproteinases (MMPs), tissue factor (TF), and inflammatory cytokines. Because these molecules are thought to contribute to plaque rupture and acute thrombosis, macrophage death in this particular context might be protective.

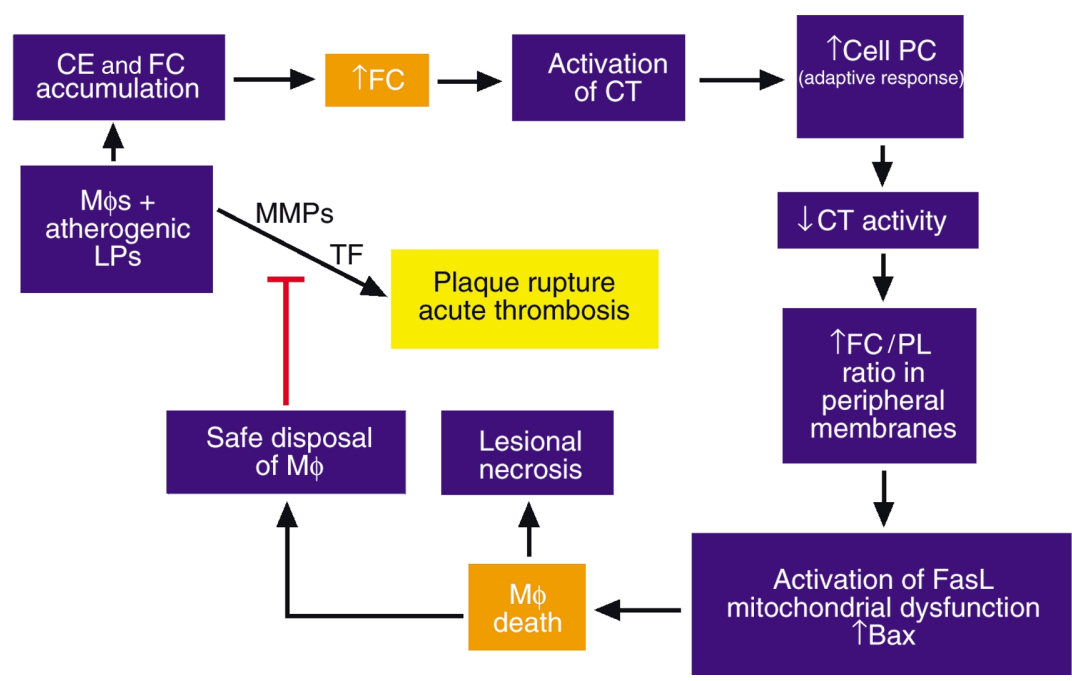


inflammation. On the other hand, death of macrophages by either necrosis or apoptosis might lead to release of cellular proteases, inflammatory cytokines, and prothrombotic molecules, which could contribute to plaque instability, plaque rupture, and acute thrombotic vascular occlusion. Indeed, necrotic areas of advanced atherosclerotic lesions are known to be associated with death of macrophages, and ruptured plaques from human lesions have been shown to be enriched in apoptotic macrophages (46).

Two mouse models have begun to shed light on the in vivo consequences of FC-induced macrophage death. In the first model, LDL receptor knockout mice were reconstituted with ACAT-1-deficient macrophages by bone marrow transplantation (47). Compared with control mice reconstituted with ACAT-1-positive macrophages, the atherosclerotic lesions of the experimental mice were larger and had increased FC content and more apoptotic macrophages. Thus, in this model, FC-induced macrophage death promotes lesion development.

The second model was designed to address the effect of preventing macrophage death in an otherwise atherosclerosis-prone mouse. By crossing a disrupted allele of $N p c 1$, in heterozygous form, into an apoE knockout background, our group produced animals with advanced atherosclerotic lesions whose macrophages were relatively resistant to FC-induced death. Control mice with normal npc1 function had large acellular areas filled with FC and macrophage debris, but not with CE. In contrast, the lesions of the mice with partial npc1 deficiency had a greater content of CE-rich macrophages (44). Because npc1 deficiency does not protect macrophages from other inducers of apoptosis, these data support the hypothesis that FC-induced death, requiring intact FC trafficking, is an important cause of lesional macrophage death in vivo. Moreover, the lesions in the death-resistant model appear more stable, but future studies with rupture-susceptible mice will be needed to substantiate this point.

In light of these recent data, the therapeutic value of ACAT inhibitors may require some further scrutiny. These agents, which are intended to block the accumulation of CE in vascular macrophages, have been proposed for the prevention or treatment of atherosclerotic vascular disease (48). Indeed, ACAT inhibitors have been shown to prevent atherosclerosis in several animal models, and one such inhibitor is currently undergoing trials in humans. The site of drug action may be key to explaining the beneficial effects of these drugs despite the apparent risk that they could further the progression of atherosclerotic lesions. First, even for ACAT-1 inhibitors, which suppress macrophage-associated enzymatic activity in vitro, the drug's ability to enter the lesion may be limited. Second, moderate suppression of ACAT activity in these cells can probably be offset by cholesterol efflux, as long as the cells have access to cholesterol acceptors such as HDL or apoA-1. ACAT-2 inhibitors, conversely, should have no direct effect on lesional macrophages and could be strongly beneficial because of their ability to suppress lipoprotein production by the intestine and - in mice - the liver (49). For these reasons, ACAT inhibitors, whether directed at one or both of these isoforms, may be less likely to precipitate macrophage death and lesional necrosis than work with ACAT-1-deficient cells might suggest.

\section{Concluding remarks and future directions}

The study of cellular cholesterol excess provides an opportunity to address a variety of important topics ranging from biophysical chemistry to intracellular signaling pathways to mechanisms of clinical disease. To begin, a proper understanding of the FC-loaded cell requires an appreciation of membrane lipid phase behavior. One must then investigate the alterations of specific enzymes and other proteins to elucidate the mechanisms of specific consequences of FC loading on cellular physiology. These alterations may result either from a direct consequence of membrane alterations or from the activation of signaling reactions that themselves are triggered by membrane alterations. Moreover, effects of FC or FC metabolites on gene expression must also be considered. Finally, the biology of the FC-loaded cell must be placed in the context of the whole tissue and organism, as demonstrated by the potential effects of the FC-loaded macrophage on atherosclerosis.

In each area, much remains to be done. While the effects of high levels of FC on the physical properties of model membranes have been studied widely in vitro, biophysical studies on excess FC in membranes of living cells have suffered from technological difficulties. New advances in fluorescence microscopy, including the use of domain-specific probes (Maxfield and Wüstner, this series, ref. 12), are beginning to close this critical gap. The consequences of cellular FC excess on specific molecules or signaling pathways are gradually entering the scientific literature, but insight into the mechanisms linking these consequences with changes in FC-induced alterations in membrane structure and possibly gene expression is lacking. For example, much has been published on the effects of cellular cholesterol depletion on raft structure and function (Simons and Ehehalt, this series, ref. 11), but very little work has been published about the effects of cellular cholesterol excess on raft biology.

The impetus for such work is related to the issues discussed immediately above, namely, the role of the FCloaded cell in organismal physiology and pathophysiology. In particular, the leading cause of death in the industrialized world is atherosclerotic vascular disease, and the cholesterol-loaded macrophage is a critical cellular component of the atherosclerotic lesion. While the atherosclerotic macrophage foam cell is typically viewed as a CE-rich cell with normal or perhaps slightly elevated levels of FC, biochemical and morphological studies have shown that progression of atherosclerosis is associated with an increase in FC and a decrease in CE in lesional macrophages. Given the potential consequences of FC loading for macrophage physiology, particularly the potential relationships between FC-induced macrophage death, lesional necrosis, and plaque rupture, one might argue that the conversion of the CEladen macrophage into an FC-loaded cell is a critical transition point in atherogenesis (Figure 3). Support for 
this concept will require much further work, particularly in vivo using genetically altered mice. Nonetheless, given the current evidence and the importance of atherosclerosis, the rationale for gaining a thorough understanding of the biology of the FC-loaded cell is clear.

1. Dietschy, J.M., Turley, S.D., and Spady, D.K. 1993. Role of the liver in the maintenance of cholesterol and low density lipoprotein homeostasis in different animal species, including humans. J. Lipid Res. 34:1637-1659.

2. Tabas, I., and Kreiger, M. 1999. Lipoprotein receptors and cellular cholesterol metabolism in health and disease. In Molecular basis of heart disease. K.R. Chien, editor. W.B. Saunders Co. New York, New York, USA. 428-457.

3. Lin, D., et al. 1995. Role of steroidogenic acute regulatory protein in adrenal and gonadal steroidogenesis. Science. 267:1828-1831.

4. Tall, A.R., Costet, P., and Wang, N. 2002. Regulation and mechanisms of macrophage cholesterol efflux. J. Clin. Invest. 110:899-904. doi:10.1172/ JCI200216391.

5. Glass, C.K., and Witztum, J.L. 2001. Atherosclerosis: the road ahead. Cell. 104:503-516.

6. Horton, J.D., Goldstein, J.L., and Brown, M.S. 2002. SREBPs: activators of the complete program of cholesterol and fatty acid synthesis in the liver. J. Clin. Invest. 109:1125-1131. doi:10.1172/JCI200215593.

7. Chang, T.Y., et al. 2001. Roles of acyl-coenzyme A:cholesterol acyltransferase-1 and -2. Curr. Opin. Lipidol. 12:289-296.

8. Björkhem, I. 2002. Do oxysterols control cholesterol homeostasis? J. Clin Invest. 110:725-730. doi:10.1172/JCI200216388.

9. Tabas, I. 1997. Free cholesterol-induced cytotoxicity. A possible contributing factor to macrophage foam cell necrosis in advanced atherosclerotic lesions. Trends Cardiovasc. Med. 7:256-263.

10. Simons, K., and Ikonen, E. 2000. How cells handle cholesterol. Science. 290:1721-1726.

11. Simons, K., and Ehehalt, R. 2002. Cholesterol, lipid rafts, and disease. J. Clin. Invest. 110:597-603. doi:10.1172/JCI200216390.

12. Maxfield, F.R., and Wüstner, D. 2002. Intracellular cholesterol transport. J. Clin. Invest. 110:891-898. doi:10.1172/JCI200216500.

13. Tabas, I. 2002. Cholesterol in health and disease. J. Clin. Invest. 110:583-590. doi:10.1172/JCI200216381.

14. Yeagle, P.L. 1991. Modulation of membrane function by cholesterol. Biochimie. 73:1303-1310.

15. Puglielli, L., et al. 2001. Acyl-coenzyme A:cholesterol acyltransferase modulates the generation of the amyloid beta-peptide. Nat. Cell Biol. 3:905-912.

16. Milhiet, P.E., Giocondi, M.C., and Le Grimellec, C. 2002. Cholesterol is not crucial for the existence of microdomains in kidney brush-border membrane models. J. Biol. Chem. 277:875-878.

17. Kellner-Weibel, G., et al. 1999. Crystallization of free cholesterol in model macrophage foam cells. Arterioscler. Thromb. Vasc. Biol. 19:1891-1898.

18. Lupu, F., Danaricu, I., and Simionescu, N. 1987. Development of intracellular lipid deposits in the lipid-laden cells of atherosclerotic lesions. A cytochemical and ultrastructural study. Atherosclerosis. 67:127-142.

19. Brown, A.J., and Jessup, W. 1999. Oxysterols and atherosclerosis. Atherosclerosis. 142:1-28.

20. Kellner-Weibel, G., et al. 1998. Effects of intracellular free cholesterol accumulation on macrophage viability: a model for foam cell death. Arterioscler. Thromb. Vasc. Biol. 18:423-431.

21. Yao, P.M., and Tabas, I. 2000. Free cholesterol loading of macrophages induces apoptosis involving the Fas pathway. J. Biol. Chem 275:23807-23813.

22. Yao, P.M., and Tabas, I. 2001. Free cholesterol loading of macrophages is associated with widespread mitochondrial dysfunction and activation of the mitochondrial apoptosis pathway. J. Biol. Chem. 276:42468-42476.

23. Ross, R. 1995. Cell biology of atherosclerosis. Annu. Rev. Physiol. 57:791-804.

24. Katz, S.S., Shipley, G.G., and Small, D.M. 1976. Physical chemistry of the lipids of human atherosclerotic lesions. Demonstration of a lesion intermediate between fatty streaks and advanced plaques. J. Clin. Invest. 58:200-211.

25. Liscum, L., and Munn, N.J. 1999. Intracellular cholesterol transport. Biochim. Biophys. Acta. 1438:19-37.

26. Lange, Y. 1998. Intracellular cholesterol movement and homeostasis. In Intracellular cholesterol trafficking. T.Y. Chang and D.A. Freeman, editors.
Kluwer Academic Publishers. Boston, Massachusetts, USA 15-27.

27. Maor, I., Mandel, H., and Aviram, M. 1995. Macrophage uptake of oxidized LDL inhibits lysosomal sphingomyelinase, thus causing the accumulation of unesterified cholesterol-sphingomyelin-rich particles in the lysosomes. A possible role for 7-ketocholesterol. Arterioscler. Thromb. Vasc. Biol. 15:1378-1387.

28. Leventhal, A.R., Chen, W., Tall, A.R., and Tabas, I. 2001. Acid sphingomyelinase-deficient macrophages have defective cholesterol trafficking and efflux. J. Biol. Chem. 276:44976-44983.

29. Reagan, J.W., Jr., Hubbert, M.L., and Shelness, G.S. 2000. Posttranslational regulation of acid sphingomyelinase in niemann-pick type C1 fibroblasts and free cholesterol-enriched chinese hamster ovary cells. J. Biol. Chem. 275:38104-38110.

30. Tangirala, R.K., Mahlberg, F.H., Glick, J.M., Jerome, W.G., and Rothblat, G.H. 1993. Lysosomal accumulation of unesterified cholesterol in model macrophage foam cells. J. Biol. Chem. 268:9653-9660.

31. Feng, B., and Tabas, I. 2002. ABCA1-mediated cholesterol efflux is defective in free cholesterol-loaded macrophages. Mechanism involves enhanced ABCA1 degradation in a process requiring full npc1 activity. J. Biol. Chem. In press.

32. Chang, T.Y., Chang, C.C.Y., and Cheng, D. 1997. Acyl-coenzyme A:cholesterol acyltransferase. Annu. Rev. Biochem. 66:613-638.

33. Moynault, A., Luciani, M.F., and Chimini, G. 1998. ABC1, the mammalian homologue of the engulfment gene ced-7, is required during phagocytosis of both necrotic and apoptotic cells. Biochem. Soc. Trans. 26:629-635.

34. Mori, M., et al. 2001. Foam cell formation containing lipid droplets enriched with free cholesterol by hyperlipidemic serum. J. Lipid Res. 42:1771-1781.

35. Tabas, I. 2000. Cholesterol and phospholipid metabolism in macrophages. Biochim. Biophys. Acta. 1529:164-174.

36. Shio, H., Haley, N.J., and Fowler, S. 1979. Characterization of lipid-laden aortic cells from cholesterol-fed rabbits. III. Intracellular localization of cholesterol and cholesteryl ester. Lab. Invest. 41:160-167.

37. Zilversmit, D.B., Shore, M.L., and Ackerman, R.F. 1954. The origin of aortic phospholipid in rabbit atheromatosis. Circulation. 9:581-585.

38. Zhang, D., et al. 2000. Macrophages deficient in CTP:Phosphocholine cytidylyltransferase-alpha are viable under normal culture conditions but are highly susceptible to free cholesterol-induced death. Molecular genetic evidence that the induction of phosphatidylcholine biosynthesis in free cholesterol-loaded macrophages is an adaptive response. J. Biol. Chem. 275:35368-35376.

39. Blom, T.S., et al. 2001. Mass spectrometric analysis reveals an increase in plasma membrane polyunsaturated phospholipid species upon cellular cholesterol loading. Biochemistry. 40:14635-14644.

40. Huster, D., Arnold, K., and Gawrisch, K. 1998. Influence of docosahexaenoic acid and cholesterol on lateral lipid organization in phospholipid mixtures. Biochemistry. 37:17299-17308.

41. Warner, G.J., Stoudt, G., Bamberger, M., Johnson, W.J., and Rothblat, G.H. 1995. Cell toxicity induced by inhibition of acyl coenzyme A: cholesterol acyltransferase and accumulation of unesterified cholesterol. J. Biol. Chem. 270:5772-5778.

42. Tabas, I., Marathe, S., Keesler, G.A., Beatini, N., and Shiratori, Y. 1996. Evidence that the initial up-regulation of phosphatidylcholine biosynthesis in free cholesterol-loaded macrophages is an adaptive response that prevents cholesterol-induced cellular necrosis. Proposed role of an eventual failure of this response in foam cell necrosis in advanced atherosclerosis. J. Biol. Chem. 271:22773-22781.

43. Formigli, L., et al. 2000. Aponecrosis: morphological and biochemical exploration of a syncretic process of cell death sharing apoptosis and necrosis. J. Cell. Physiol. 182:41-49.

44. Zhang, D., et al. 2001. Niemann-Pick C heterozygosity confers marked protection from macrophage death and lesional necrosis. Circulation. 104:II-45. (Abstr.)

45. Kockx, M.M. 1998. Apoptosis in the atherosclerotic plaque: quantitative and qualitative aspects. Arterioscler. Thromb. Vasc. Biol. 18:1519-1522.

46. Mitchinson, M.J., Hardwick, S.J., and Bennett, M.R. 1996. Cell death in atherosclerotic plaques. Curr. Opin. Lipidol. 7:324-329.

47. Fazio, S., et al. 2001. Increased atherosclerosis in LDL receptor-null mice lacking ACAT1 in macrophages. J. Clin. Invest. 107:163-171.

48. Brown, W.V. 2001. Therapies on the horizon for cholesterol reduction. Clin. Cardiol. 24:III24-III27.

49. Buhman, K.K., et al. 2000. Resistance to diet-induced hypercholesterolemia and gallstone formation in ACAT2-deficient mice. Nat. Med. 6:1341-1347. 